\title{
Au intensity enhancement for RHIC
}

\author{
S.Y. Zhang, H. Huang
}

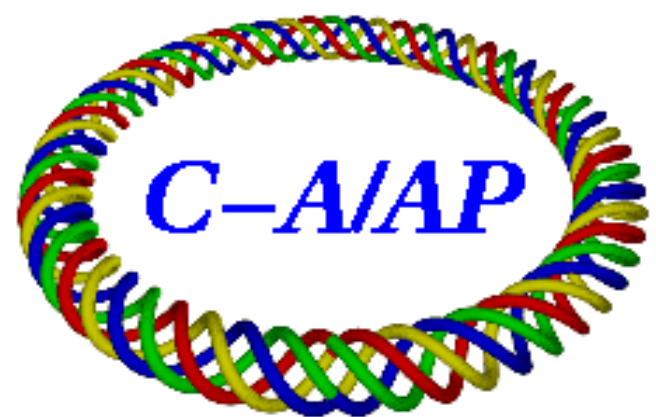

\section{Collider-Accelerator Department Brookhaven National Laboratory Upton, NY 11973}

\section{U.S. Department of Energy Office of Science, Office of Nuclear Physics}

Notice: This document has been authorized by employees of Brookhaven Science Associates, LLC under Contract No. DE-SC0012704 with the U.S. Department of Energy. The United States Government retains a nonexclusive, paid-up, irrevocable, world-wide license to publish or reproduce the published form of this document, or allow others to do so, for United States Government purposes. 


\title{
Au Intensity Enhancement for RHIC
}

\author{
S.Y. Zhang and H. Huang
}

December 9, 2015

\section{Introduction}

Very successful $100 \mathrm{GeV} / \mathrm{n}$ Au-Au run 2014 has demonstrated that more than $90 \%$ beam loss is due to luminosity production (burn-off), which leaves the enhancement of RHIC Au intensity as dominated target for further luminosity improvement.

In past runs, the performance of EBIS, Booster, and AGS has been progressed incrementally, but not drastically. The main focus of RHIC intensity enhancement is therefore a further bunch merge. Under existing machine conditions, the Au bunch merge of 6-3-1 in AGS becomes the first try, and in run 2015, it has been briefly tested [1].

The test opened door for RHIC Au intensity enhancement, where the 6-3-1 scheme is targeting $50 \%$ intensity improvement, and other schemes, such as 8-4-1, upgrade permitted, may improve more. The benefit and importance to RHIC programs are self-explained:

1. In next $100 \mathrm{GeV} / \mathrm{n} \mathrm{Au-Au}$ run 2016, 50\% intensity enhancement implies more than doubled luminosity. Significant benefit also exists for p-Au and d-Au runs.

2. In low energy BES2 runs, the Au intensity is an important aspect, in addition to cooling.

3. Same is true for e-Au luminosity enhancement in eRHIC.

In this article, possible improvement related to the AGS 6-3-1 bunch merge test is presented. After that, relevant issues for beams with larger longitudinal emittance at RHIC are discussed.

The 6-3-1 bunch merge test in run 2015 resulted in 10\% intensity improvement in RHIC injection, not $50 \%$. Therefore, the mechanism of the beam loss, mostly around AGS early acceleration, is a first focus of discussion.

Possible beam loss mechanism is as follows:

1. The AGS accelerating bucket area is limited at the early acceleration.

2. Space charge effect around the same period of time is not trivial, and this effect would be more important along with intensity push.

3. Beam momentum spread related dispersion effect at the same period of time, it is not insignificant. 
4. Squeezing beam into the accelerating bucket, which produced 'baby' bunches. This loss in 6-3-1 test became more significant.

It is noted that in the test the beam longitudinal emittance at RHIC injection is $0.58 \mathrm{eVs} / \mathrm{n}$, not the expected $0.75 \mathrm{eVs} / \mathrm{n}$. Meanwhile, the bucket area at the early AGS acceleration, with the current harmonic and voltage on the accelerating cavity, is limited at about $0.6 \mathrm{eVs} / \mathrm{n}$.

We focus on accelerating bucket area limit first. Should other mechanism be dominant, the longitudinal emittance at the RHIC injection could be larger than $0.58 \mathrm{eVs} / \mathrm{n}$.

With this consideration, possible schemes to improve the AGS accelerating beam loss are as follows:

1. Reducing RF harmonic number is the most effective one to enlarge the bucket area. Currently, the harmonic number is 12 , should it be 11, 10, then with the same RF voltage, the bucket area would be $14 \%$ and $31 \%$ larger, respectively.

2. If the AGS injection energy can be raised, the bucket area is enlarged.

3. If an early acceleration before squeezing is possible, then the most tight limit of the existing accelerating cavity can be bypassed.

These possibilities will be discussed, along with the space charge, dispersion, and squeezing losses. There are many issues associated with each scheme, some of that might be game stoppers, so the discussion presented here is the first step of studies.

With the better 6-3-1 bunch merge, the longitudinal emittance should be larger, and meanwhile the RHIC injection time will also be longer. The IBS induced emittance growth at the injection and ramp needs an attention.

In numerous RHIC low energy $\mathrm{Au}$ runs, it has demonstrated that the IBS growth rate at lower energy is much weaker than that at high energy. With the modified IBS model, which is based on RHIC observations of many low energy Au runs, the scenario of the IBS growth with more bunch merge is presented. The emittance growth is larger, but tolerable.

The larger longitudinal emittance also raise concern for beam rebucketing. In RHIC run 2010, rather large longitudinal emittance had been handled by using higher voltage on 197 $\mathrm{MHz}$ cavity, the rebucketing had proceeded well, and luminosity had improved. Therefore, the rebucketing for more bunch merge, which is actually with smaller longitudinal emittance than run 2010, should be manageable.

With larger longitudinal emittance and the required higher rebucketing voltage, the beam momentum spread at store will be larger. The beam lifetime would be affected, which has been demonstrated in run 2010. Note that then the IBS suppression lattice was used, which had very limited dynamic aperture. It will be of interest to see the beam lifetime with the nominal lattice, which has much larger dynamic aperture.

Finally, the strategy of the RHIC Au luminosity improvement with the intensity enhancement will be discussed. It is suggested that the intensity enhancement with more bunch merge should be focused on first, due to its quadratic relation with the luminosity. Along with this effort, the longitudinal emittance could be incrementally improved, from EBIS to RHIC. 


\section{Reducing harmonic of the accelerating cavity}

Reducing accelerating cavity harmonic is perhaps the most effective way to get larger bucket, due to the fact of

$$
A_{b k s} \propto \frac{1}{h^{3 / 2}}
$$

where $h$ is the harmonic number, and $A_{b k s}$ is the stationary bucket area. The moving bucket area, which depends not only on the stationary bucket but also the magnetic ramping, is smaller, but not too much. The relation of bucket area to harmonic is the same.

The most crucial factor related to harmonic number is the effective voltage received by beam, which is depending on the ferrite frequency response.

In Table 1, harmonic number 12, 11, and 10 are shown for the bucket area and the frequency, at the AGS injection energy.

\begin{tabular}{|c|c|c|c|c|c|}
\hline$h$ & 12 & 11 & $\Delta / 12$ & 10 & $\Delta / 12$ \\
\hline$f_{r f}, M H z$ & 1.956 & 1.793 & & 1.630 & \\
\hline$A_{b k s}, e V s / n$ & 0.665 & 0.758 & $14 \%$ & 0.875 & $31 \%$ \\
\hline
\end{tabular}

Table 1: RF frequency and bucket area for different harmonic number at the AGS injection energy, all with the RF voltage of $200 \mathrm{kV}$.

To squeeze beam into the accelerating bucket with larger area is easier, and therefore less "baby" bunches would be produced for lower harmonics.

It is also of interest to know the effects of space charge and dispersion with the lower harmonics. For that the bunch intensity of $2 \times 10^{9}$, the longitudinal emittance of $0.6 \mathrm{eVs} / \mathrm{n}$, the dispersion of $2 \mathrm{~m}$, and the RF voltage of $200 \mathrm{kV}$ are used to get the bunch length, momentum spread, dispersion effect, and space charge tune shift, which are shown in Table 2.

\begin{tabular}{|c|c|c|c|c|c|}
\hline$h$ & 12 & 11 & $\Delta / 12$ & 10 & $\Delta / 12$ \\
\hline$\tau_{\ell}, n s$ & 350 & 358 & & 367 & \\
\hline$d p / p, \%$ & 0.550 & 0.539 & & 0.526 & \\
\hline$\Delta x_{D}, m m$ & 11.0 & 10.8 & $-2 \%$ & 10.5 & $-4 \%$ \\
\hline$\Delta \nu_{\text {inc }}, \%$ & 22.8 & 22.3 & $-2 \%$ & 21.8 & $-4 \%$ \\
\hline
\end{tabular}

Table 2: Here $\tau_{\ell}$ is the bunch length, $d p / p$ is the momentum spread, $\Delta x_{D}$ is the dispersion effect, and $\Delta \nu_{i n c}$ is the incoherent space charge tune shift. With lower harmonics, both the dispersion and space charge effects are reduced.

It is noted that the nonlinearity of RF focusing is neglected, therefore, the beam momentum spread shown here is a little larger than it should be, which nevertheless is good for comparison.

The space charge incoherent tune shift is proportional to intensity, for example, with bunch intensity of $3 \times 10^{9}, \Delta \nu_{i n c}$ with harmonic 12 the space charge tune shift is $34.2 \%, 50 \%$ larger than that shown in Table 2 . This space charge tune shift is likely to cause beam loss. 
The harmonic number 11 should be doable with the known $1.8 \mathrm{M} \mathrm{Hz}$ frequency range of the existing accelerating cavity. This harmonic can be used for 6-3-1 merge, but not the 12-6-2 merge [2]. For harmonic 10, the effective voltage might be lower.

The ferrite frequency response of the accelerating cavity is of interest to know, a measurement of frequency response of the ferrite and the associated control circuits is suggested.

On the other hand, even with lower voltage, the bucket area, the space charge, the dispersion, and also the squeezing may still benefit with lower harmonics. In Table 3, these are compared with $175 \mathrm{kV}$ at harmonic 11, and $150 \mathrm{kV}$ at harmonic 10 .

\begin{tabular}{|c|c|c|c|c|c|}
\hline$h$ & 12 & 11 & $\Delta / 12$ & 10 & $\Delta / 12$ \\
\hline$V, k V$ & 200 & 175 & & 150 & \\
\hline$A_{b k s}, e V s / n$ & 0.665 & 0.709 & $7 \%$ & 0.757 & $14 \%$ \\
\hline$\tau, n s$ & 350 & 370 & & 394 & \\
\hline$d p / p, \%$ & 0.550 & 0.521 & & 0.489 & \\
\hline$\Delta x_{D}, m m$ & 11.0 & 10.4 & $-5 \%$ & 9.78 & $-11 \%$ \\
\hline$\Delta \nu_{\text {inc }}$ & 22.8 & 21.6 & $-5 \%$ & 20.3 & $-11 \%$ \\
\hline
\end{tabular}

Table 3: Same as Table 2 except the lower voltage for lower harmonics. Bucket area increased less, but space charge and dispersion effects reduced more, which are due to longer bunches with lower RF voltages.

Once the ferrite frequency response of the accelerating cavity is known, one may choose the better settings to reduce beam loss in accelerating, with consideration of bucket area, space charge, dispersion and squeezing.

\section{$3 \quad$ Energy factor of the accelerating cavity}

At higher energy, the accelerating cavity has larger bucket area, and the beam space charge and dispersion effects are also reduced.

With respect to the concerns of beam loss at the early AGS acceleration, there are two ways to do this:

1. To raise the AGS injection energy, and for that the Booster extraction, BtA, and AGS injection all need to be modified. In 1997, the AGS proton injection energy was raised from $1.54 \mathrm{GeV}$ to $1.9 \mathrm{GeV}$, the AGS space charge effect was reduced by more than $30 \%$, and the AGS injection loss was reduced more than $10^{13}$ protons for each AGS extraction of $6 \times 10^{13}$ protons. There are many issues in this direction for Au operations, and it would be of interest to know, especially if there are game stoppers. If AGS injection energy could be raised, then the stripping efficiency can be improved [2], which is also of interest to know.

2. To accelerate before squeezing. Currently, the bunch is merged into the harmonic 4 and harmonic 8 cavities, which combined has large area. To squeeze into the smaller harmonic 
12 accelerating cavity causes "baby" bunches and also beam loss. The harmonic 4 and harmonic 8 cavities are not prepared with the accelerating capability, but it is also of interest to know how much can be done, or if an upgrade is possible.

These works may require upgrade. Therefore, the relevant parameters with the higher energy are provided in Table 4, as a wish list.

\begin{tabular}{|c|c|c|c|c|c|}
\hline$\Delta \beta / \beta$ & $0 \%$ & $10 \%$ & $\Delta / 0 \%$ & $20 \%$ & $\Delta / 0 \%$ \\
\hline$\beta$ & 0.439 & 0.483 & & 0.527 & \\
\hline$E_{k}, G e V / n$ & 0.105 & 0.132 & & 0.161 & \\
\hline$A_{b k s}, e V s / n$ & 0.665 & 0.692 & $4.1 \%$ & 0.724 & $8.9 \%$ \\
\hline$\tau_{\ell}, n s$ & 350 & 312 & & 280 & \\
\hline$d p / p, \%$ & 0.550 & 0.497 & & 0.450 & \\
\hline$\Delta x_{D}, m m$ & 11.0 & 9.9 & $-10 \%$ & 9.0 & $-18 \%$ \\
\hline$\Delta \nu_{\text {inc }}, \%$ & 22.8 & 20.1 & $-12 \%$ & 17.7 & $-22 \%$ \\
\hline
\end{tabular}

Table 4: Higher energy parameters for the RF bucket, space charge and dispersion effects. Harmonic number $h$ is 12 , bunch intensity is $2 \times 10^{9}$, longitudinal emittance is $0.6 \mathrm{eVs} / \mathrm{n}$, RF voltage is $200 \mathrm{kV}$

\section{$4 \quad$ IBS effect with more bunch merges}

With more bunch merge, the longitudinal emittance would be larger. For 6-3-1, or 12-6-2 [2] schemes, the longitudinal emittance at RHIC injection should be $50 \%$ higher, which says that without AGS bucket limit, beam of $0.75 \mathrm{eVs} / \mathrm{n}$ is expected at RHIC injection. Currently, the longitudinal emittance at the RHIC injection is about $0.5 \mathrm{eVs} / \mathrm{n}$.

Also, with the 6-3-1 scheme, the RHIC injection time would be extended from the current 5 minutes to 10 minutes.

In [3], the IBS effect on the longitudinal emittance growth at RHIC has been discussed, which is based on RHIC low energy runs at 9.8, 13.5, 19.5, 31.5 GeV/n, compared with 100 $\mathrm{GeV} / n$ runs.

The longitudinal growth rate in that IBS formula has a dependence on energy,

$$
\tau_{\|}^{-1} \propto \frac{1}{\beta^{3 / 2} \gamma^{3 / 2} \tau_{\ell}(d p / p)^{2}}=\eta^{1 / 4} \beta^{3 / 2} \gamma^{1 / 4}
$$

With the energy factor not only in $\beta, \gamma$, but also in bunch length, $\tau_{\ell}$, and momentum spread, $d p / p$, this formula shows very weak dependence on energy. In specific, with this formula, the IBS longitudinal growth rate at the RHIC full energy is only $18 \%$ larger than that at the injection.

A scaler modification of the IBS model on energy is suggested in [3], based on observations in numerous RHIC runs at different energies, where the longitudinal IBS growth rate at the RHIC injection is only about $10 \%$ of that at the top energy. 

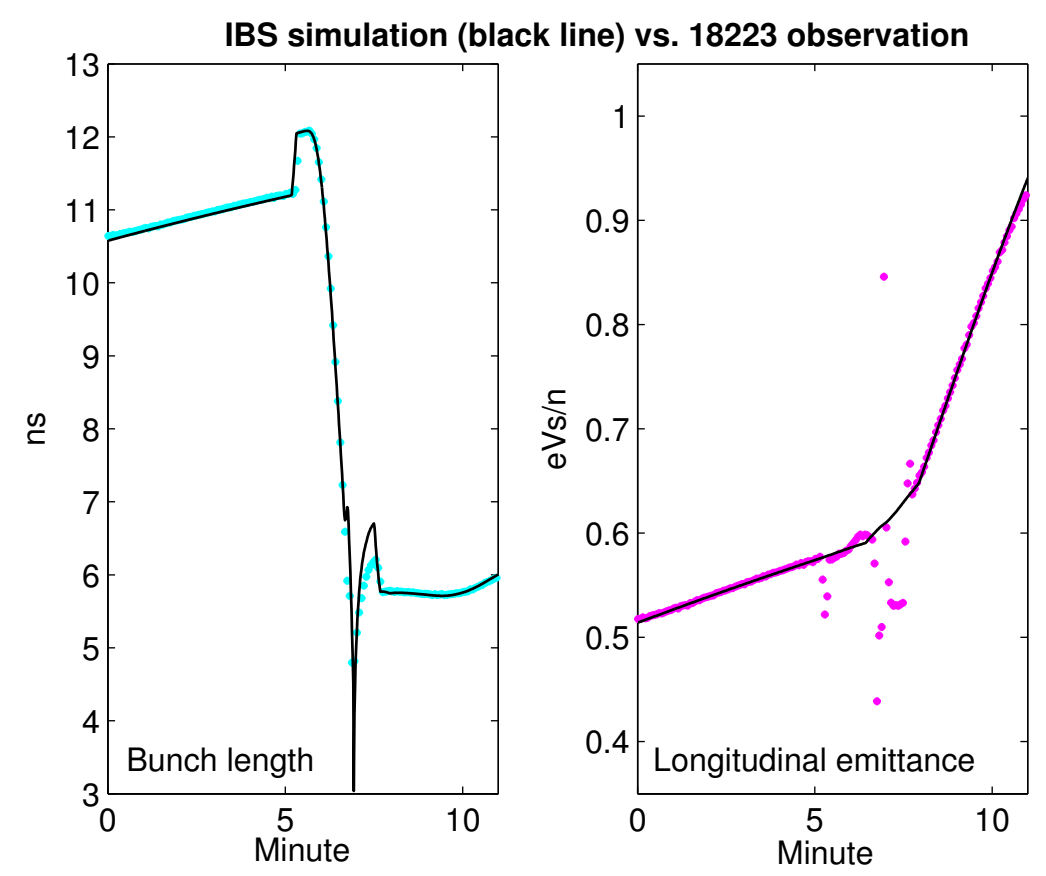

Figure 1: The energy scaler modified IBS model on RHIC bunch length and longitudinal emittance growth are compared with the observation of Fill 18223. The longitudinal growth rate at the injection energy, before 5.5 minute, is much weaker than that at the high energy.

In Fig.1, the modified IBS model is applied to the WCM observation of Fill 18223, where the acceleration takes place at 5.5 minute and finished at 11 minute. It can be seen that before 5.5 minute, at the injection energy, the IBS induced longitudinal emittance growth is not strong.

Based on this model, the possible scenario of the 6-3-1 bunch merge at RHIC injection and ramp is shown in Fig.2. The injected bunch is with the longitudinal emittance of $0.75 \mathrm{eVs} / \mathrm{n}$, the intensity is $3 \times 10^{9}$, the RHIC injection time is extended to 10 minutes, whereas other machine conditions are the same as Fill 18223, including the RF voltage and other ramping parameters.

In brief, the beam injected into RHIC with the longitudinal and transverse emittance of 0.75 $\mathrm{eVs} / \mathrm{n}$ and $10 \pi \mu \mathrm{m}$, in 10 minutes of the injection, it becomes $0.91 \mathrm{eVs} / \mathrm{n}$ and $11.3 \pi \mu \mathrm{m}$, and at the end of ramping, it is $1.40 \mathrm{eVs} / \mathrm{n}$ and $14.0 \pi \mu \mathrm{m}$.

The extended 5 minutes at the injection induces about $10 \%$ more longitudinal emittance growth, which is not significant.

The enlarged longitudinal emittance of $0.75 \mathrm{eVs} / n$ is ended up with $1.40 \mathrm{eVs} / \mathrm{n}$ at the top energy. Should it be the current $0.50 \mathrm{eVs} / \mathrm{n}$ at the injection, it would be $1.21 \mathrm{eVs} / \mathrm{n}$. The overall growth is $87 \%$ for $0.75 \mathrm{eVs} / \mathrm{n}$ and $142 \%$ for $0.50 \mathrm{eVs} / \mathrm{n}$. The higher growth rate with the initial lower $0.50 \mathrm{eVs} / \mathrm{n}$ is due to the higher beam density.

In overall, the end longitudinal emittance of $1.40 \mathrm{eVs} / n$ is larger than that in the current operations, typically around $1.1 \mathrm{eVs} / \mathrm{n}$. 

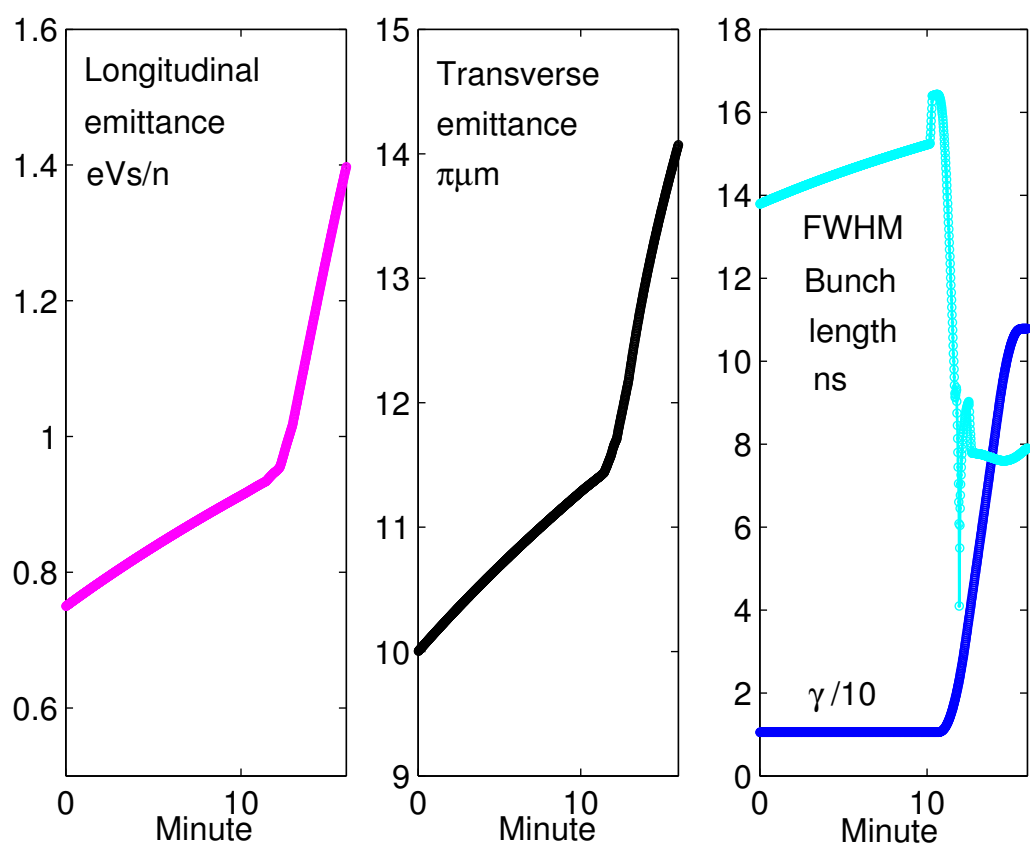

Figure 2: The possible scenario of the 6-3-1 bunch merge, without AGS bucket area limit. The RHIC injected beam has the longitudinal emittance of $0.75 \mathrm{eVs} / \mathrm{n}$, and the RHIC injection time is 10 minutes.

\section{$5 \quad$ Rebucketing and other issues}

Most concerned issue for larger longitudinal emittance is the rebucketing. The first question is how to handle the larger longitudinal emittance, say $1.40 \mathrm{eVs} / \mathrm{n}$, at the rebucketing.

In the entire $100 \mathrm{GeV} / \mathrm{n}$ run 2010, there was a longitudinal emittance blow-up at the RHIC transition. The rebucketing was affected, but the situation is well handled with higher RF voltage: the $197 \mathrm{MHz}$ cavity voltage had been increased to more than $4 \mathrm{MV}$.

In Fig.3, for Golden fill 11858, the observed bunch length, longitudinal emittance, and bunch profile at the rebucketing are shown. The beam injected into RHIC with the longitudinal emittance of $0.5 \mathrm{eVs} / \mathrm{n}$, and the emittance is jumped at the transition, reaching $2.2 \mathrm{eVs} / \mathrm{n}$ before the rebucketing.

Dealing with the larger emittance, high RF voltage of the $197 \mathrm{MHz}$ cavities was applied for the rebucketing. For fill 11858, the voltage is actually $4.4 \mathrm{MV}$.

The rebucketing itself is comparable to run 2011 and run 2014, where the longitudinal emittance at the rebucketing is much smaller, since the transition emittance blow-up have been fixed.

With larger longitudinal emittance and the required higher rebucketing voltage, the beam momentum spread in store will be larger. Therefore, the second question is the beam lifetime in store.

The beam momentum spread with 4.4 $M V$ rebucketing voltage in run 2010 is $0.16 \%$. As a comparison, in run 2011, without longitudinal emittance blow-up at the transition, the lon- 

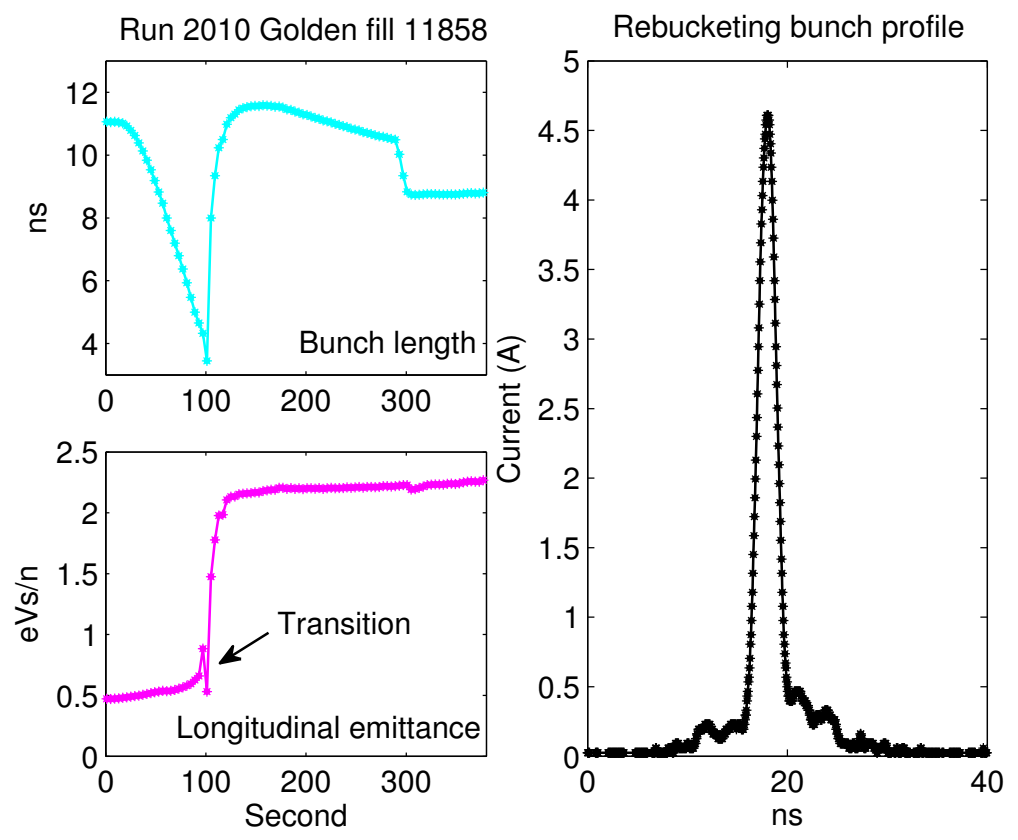

Figure 3: The bunch length, longitudinal emittance and the bunch profile after rebucketing for Golden fill 11858 in run 2010. The longitudinal emittance had a blow-up at the RHIC transition, therefore, it is $2.2 \mathrm{eVs} / \mathrm{n}$ at the rebucketing. High RF voltage of $4.4 \mathrm{MV}$ is used to handle the rebucketing.

gitudinal emittance at the rebucketing is around $1.1 \mathrm{eVs} / \mathrm{n}$, and mostly $2.5 \mathrm{MV}$ is used for rebucketing. The beam momentum spread in store is $0.12 \%$.

For 5 Golden fills in run 2010, the early luminosity (0.5 hour after transition) is $34.6 \times$ $10^{26} \mathrm{~cm}^{-2} \mathrm{~s}^{-1}$, and the average luminosity in next 3 hours is $25.3 \times 10^{26} \mathrm{~cm}^{-2} \mathrm{~s}^{-1}$, which is $73 \%$ of the peak.

In run 2011 , the early luminosity is raised to $46.6 \times 10^{26} \mathrm{~cm}^{-2} \mathrm{~s}^{-1}$, due to the higher intensity. The average luminosity is $35.6 \times 10^{26} \mathrm{~cm}^{-2} \mathrm{~s}^{-1}$, which is $76 \%$ of the peak.

For run 2010 and run 2011, the difference of the beam longitudinal emittance at the rebucketing is a factor of $2(2.2 \mathrm{eVs} / n$ vs. $1.1 \mathrm{eVs} / n)$, the difference of the rebucketing voltage is large ( $4 \mathrm{MV}$ vs. $2.5 \mathrm{MV}$ ), and therefore the difference of beam momentum spread in store is large. Nevertheless, all of these have not played a very significant role in terms of the overall luminosity. The most critical factor of the luminosity improvement in run 2011 is the higher bunch intensity.

Considering the coming run 2016, it is reminded that in run 2010 the IBS suppression lattice was used, which has a rather limited dynamic aperture [4]. Even with the much larger longitudinal emittance, much higher rebucketing voltage, and the IBS lattice, the Au run 2010 still achieved reasonable luminosity improvement.

Finally, the strategy of RHIC Au luminosity improvement is worth a brief discussion.

RHIC history of luminosity improvement has demonstrated that the intensity improvement has often, if not always, dominated the show. For the current Au bunch merge, this may also 
be the case.

Due to the quadratic relation to the luminosity, the intensity enhancement should be focused on first.

Meanwhile, other issues such as the longitudinal emittance improvement should be performed, most importantly at the injector chain, from EBIS, Booster, to AGS.

Without intensity push, any longitudinal emittance improvement can only take place for lower intensity beams, which is not very useful for RHIC luminosity enhancement. Only the emittance control at higher intensity is really needed for RHIC program.

The $\mathrm{Au}$ intensity enhancement should be focused on in the coming years, for luminosity improvement of RHIC heavy ion run now, and BES2 run 2019-2020, Au-Au/p-Au run 20212022, and e-Au in eRHIC.

\section{References}

[1] C. Gardner, "Gold ion intensities in the RHIC injector chain", RHIC Retreat, 2015.

[2] K. Zeno, private communications, 2015.

[3] S.Y. Zhang, "RHIC Au beam in run 2014", C-A/AP 529, 2014.

[4] S.Y. Zhang, "Comments on momentum aperture of $100 \mathrm{GeV} / \mathrm{n}$ Au runs in RHIC", C-A/AP 437, 2011. 